\title{
Fowlpox-NY-ESO-1 Vaccine
}

National Cancer Institute

\section{Source}

National Cancer Institute. Fowlpox-NY-ESO-1 Vaccine. NCI Thesaurus. Code C48465.

A cancer vaccine consisting of a recombinant fowlpox virus vector encoding an immunogenic peptide derived from the cancer-testis antigen NY-ESO-1, an antigen found in normal testis and various tumors, including bladder, breast, hepatocellular, melanoma, and prostate cancers. Vaccination with NY-ESO-1 peptide vaccine may stimulate the host immune system to mount a humoral and cytotoxic T lymphocyte (CTL) response against tumor cells expressing NY-ESO-1 antigen, resulting in tumor cell lysis. ( $\mathrm{NCl05)}$ 